\title{
Neck Maintenance Post Facelift using Ulthera DeepSEE Technology
}

\section{Farhan Taghizadeh*, Amber R Howard, Alicia Lebowitz, Patrick Ramirez and Brittney Garcia}

Lifestyle Lift, Albuquerque, NM, USA

\begin{abstract}
Background: As face lifts have become more popular with time, there has also been growing interest to find a technique that is less abrasive than the original. Research found the layer that needed to be targeted was the Superficial Musculo-Aponeurotic System (SMAS). The difficulty then became discovering a technique that only affected the SMAS, and not any of the surrounding tissue.
\end{abstract}

Methods: This study included 13 patients, who had prior deep-SMAS rhytidectomies at least 5 months prior and desired further maintenance. The Five Plus Ulthera protocol was used. Pictures were obtained prior to the treatment, one week and five months after treatment. All photos were reviewed by an independent board of plastic surgeons on the Global Aesthetic Improvement Scale. Each patient completed a survey based on pain and comfort levels 24 hours, one week, one month, and five months post treatment.

Results: There are 11 women and 2 men participating in the study with ages ranging from 54-75. Over all three intervals the GAIS average score was 3.44 , the comfort level increased and the pain assessment decreased. At 24 hours after treatment, two patients said they would not recommend or receive the treatment again however, by one week and 30 days there were no longer any patients that would not recommend or receive the treatment again. Instead there were four that would recommend and repeat the treatment, three that would repeat the procedure but not recommend it and three that were unsure of either.

Discussion: One of the most prominent issues with facelift is the maintenance of the integrity of the facelift over time. Desire has also increased for a non-invasive procedure that has positive, noticeable results in reducing rhytids and increasing elasticity. The study focused on DeepSEE ultrasound technology as being a viable Powered by Editorial Manager ${ }^{\circledR}$ and Production Manager ${ }^{\circledR}$ from Aries Systems Corporation option to fulfill these needs.

Keywords: Rhytidectomies; Superficial musculo-aponeurotic system (SMAS); Ultrasound treatment

\section{Introduction}

While the face lift became a popular surgical technique to eliminate rhytides in the aging skin, a large amount of effort was put into technological advances to develop a technique that was not so abrasive [1]. Certain patients, for example those with less severe wrinkles, those who did not want a surgical procedure done, or those who could not afford such a recovery time, desired such a treatment that could address their needs [2]. To this end, much research and money were put into devices such as monopolar and dipolar radio frequency devices but to no avail [3]. It was determined that such accuracy was required so that the layer known as the Superficial Musculo-Aponeurotic System (SMAS), which is responsible for being the most contractive muscle layer under the skin and thus causing the most rhytide elimination, must be targeted [4-9]. Not only was it determined that this layer which lies $3-5 \mathrm{~mm}$ below the skins surface to be the most crucial target but it was also found that if any of the other surrounding tissue was simultaneously subjected to the treatment then quite adverse effects could be of consequence [1].

With all of these considerations, the development of the Ulthera system is one of the more promising treatments on the market today [10]. With its long history of experimentation in targeting tumor masses at varying depth under the skin's surface [11-26], ablative ultrasound has been shown to be an effective tool in specifically targeting the SMAS while delivering quick, short burst of energy causing small and precise packets of thermal injury to the designated tissue [27]. The ultrasound waves infiltrates into the tissue causing quick vibration of the molecules. The result of this vibration is thermal injury of the focus site created by the friction of colliding molecules. The depth of penetration is inversely proportional to the frequency of the wave. A higher frequency used, results in shallow penetration while a lower frequency delivers a deeper penetration. The Ulthera device, along with a unique hand piece delivers a short pulse duration combined with low energy allowing for localization of the resulting thermal injury. Not only can the device deliver such precision in such a non-surgical manner, but it also has the advantage of the ultrasound technology which can image the target area before treatment [10]. For real-time imaging, the Ulthera device uses lower energy combined with longer pulse duration. Ultimately both the image and targeting of selected tissue with energy can be accomplished with the same hand piece. Previous studies have shown that the Ulthera device can create localized thermal injury in sub dermal soft tissue while maintaining preservation to adjacent tissues and structures.

Conversely, this ultrasound treatment will most likely not replace the original face lift procedure. It should be noted that these technologies are less efficacious than traditional surgical procedures $[28,29]$. However, after rhytidectomy, one common outcome continues to be skin laxity and there remains debate about which techniques can mitigate this regression [30,31]. Most debate centers on techniques to best address the SMAS during facelifts. In this study, we investigated the ability of ablative ultrasound to target the SMAS in a manner that is less invasive and allows for the possibility of continued tightening and reduced recidivism and laxity. Being the only device that targets deep tissue in a non-surgical manner the primary objective of this study was to determine whether ultrasound technology can be adopted

*Corresponding author: Farhan Taghizadeh, 2440 Louisiana Blvd NE Suite 550 Albuquerque, NM 87110, USA, Tel: 505-872-0074; Fax: 505-872-0058; E-mail: ftaghizadeh@lifestylelift.com

Received March 01, 2014; Accepted April 20, 2014; Published April 28, 2014

Citation: Taghizadeh F, Howard AR, Lebowitz A, Ramirez P, Garcia B (2014) Neck Maintenance Post Facelift using Ulthera DeepSEE Technology. Surgery Curr Res 4: 187. doi:10.4172/2161-1076.1000187

Copyright: (ㅇ 2014 Taghizadeh F, et al. This is an open-access article distributed under the terms of the Creative Commons Attribution License, which permits unrestricted use, distribution, and reproduction in any medium, provided the original author and source are credited. 
as a routine minimally invasive facelift maintenance tool. To our knowledge this study includes one of the first that apply the Ulthera system to facial tissues for further maintenance.

\section{Methods}

In our study there were 13 patients that had prior deep-SMAS rhytidectomies at least five months prior and desired further maintenance. All patients were consented for the procedure. Topical numbing cream of $7 \%$ lidocaine, $7 \%$ tetracaine was used. The Five Plus Ulthera protocol was utilized on all patients who comprise of using a DS 4.5 on the midface with settings $31,31,15,18,15,11$ and DS 3.0 on settings $28,28,18,15,11$. Pre-treatment photos were taken to serve as a baseline. Photos were taken using the Vectra ${ }^{\star}$ 3D imaging system by Canfield Scientific Inc. (Fairfield, NJ). Following the Five Plus Ulthera protocol, post-treatment photos were obtained at one week and 5 months. Pre- and post-treatment photographs were printed out and organized side by side to be reviewed by an independent board of plastic surgeons on the following scales: the Global Aesthetic Improvement Scale. The baseline, one week and 5 month postoperative photographs were individually given a score on the General Aesthetic Improvement Scale. These scores were organized in excel spreadsheet where the baseline scores could be compared to the one week and 5 month scores and improvements could be documented.

Each of the 13 patients was given an identical survey at 24 hours, one week, one month and 5 months post-treatment. The survey retrospectively asked patients to rate their pain and comfort level from $0-10,0$ being no pain and 10 being the worst pain, during the procedure as well as their opinion of the result of the procedure. Additional questions such as 'Would you recommend Ulthera to a friend?' and 'Would you have Ulthera treatment again?' were asked to qualitatively determine whether the pain and comfort level during the course of treatment affected their view of the treatment as a whole. The patients' recollection of their pain and comfort scores at 24 hours, 7 days, and 30 days were averaged and trends were recorded.

\section{Results}

In this study, 11 women with a mean age of 63 (Range 54-75, SD \pm 6.6) received treatment with Ulthera DeepSEE ${ }^{\text {rax }}$ technology. All subjects had undergone deep-SMAS rhytidectomy with a mean time of 10.6 months (Range 4-35 months, $\mathrm{SD} \pm 8.25$ months) prior to their treatment with Ulthera. At the 24 hour assessment period, patients were surveyed for their pain during the treatment. The mean pain score was 6 (Range $2-10, S D \pm 2$ ). At this time a survey was also done on the patient's comfort during the treatment. The mean comfort score was 6.9 (Range $3-10, S D \pm 2.5$ ). The patients were also asked whether they would recommend the procedure to a friend and if they would receive treatment again. There were 2 patients that responded 'no' they would not receive this treatment again or recommend this treatment to others while 7 patients responded 'yes' they would recommend this treatment as well as receive this treatment themselves again. Lastly, 3 patients were unsure of whether they would want to recommend this treatment or receive it again. Furthermore, at 24 hours the patients were rated by the physician on the Global Aesthetic Improvement Scale (GAIS) (Figure 1). After 24 hours, the mean GAIS score of the patients given by the physician was a 3.55 (Range $1-5, \mathrm{SD} \pm 0.75$ ) denoting a slight improvement. An assessment was administered again one week after the treatment. At this time the survey number fell to ten patients. The mean GAIS score was 3.33 , mean recall of pain score was 5 , and the mean recall of comfort score was 6.8. Four patients said that they would both recommend the treatment to others as well as receive this treatment again. Three patients said they would repeat the procedure themselves, but not recommend it to others. Lastly, three patients were not sure of whether they would want to recommend this treatment to others or receive it themselves again. The physician GAIS score at 30 days was 3.33. Retrospective pain assessment score average was 4.66, while retrospective comfort score average was a 7.66. All patients' responses as to whether they would recommend this treatment or receive it themselves were the same at 30 days as they were at one week. Lastly, patients were asked to rate their improvement after 30 days using the GAIS rankings. The self-assessment mean rating was 3.25 (Range 3-4, $\mathrm{SD} \pm .25$ ).
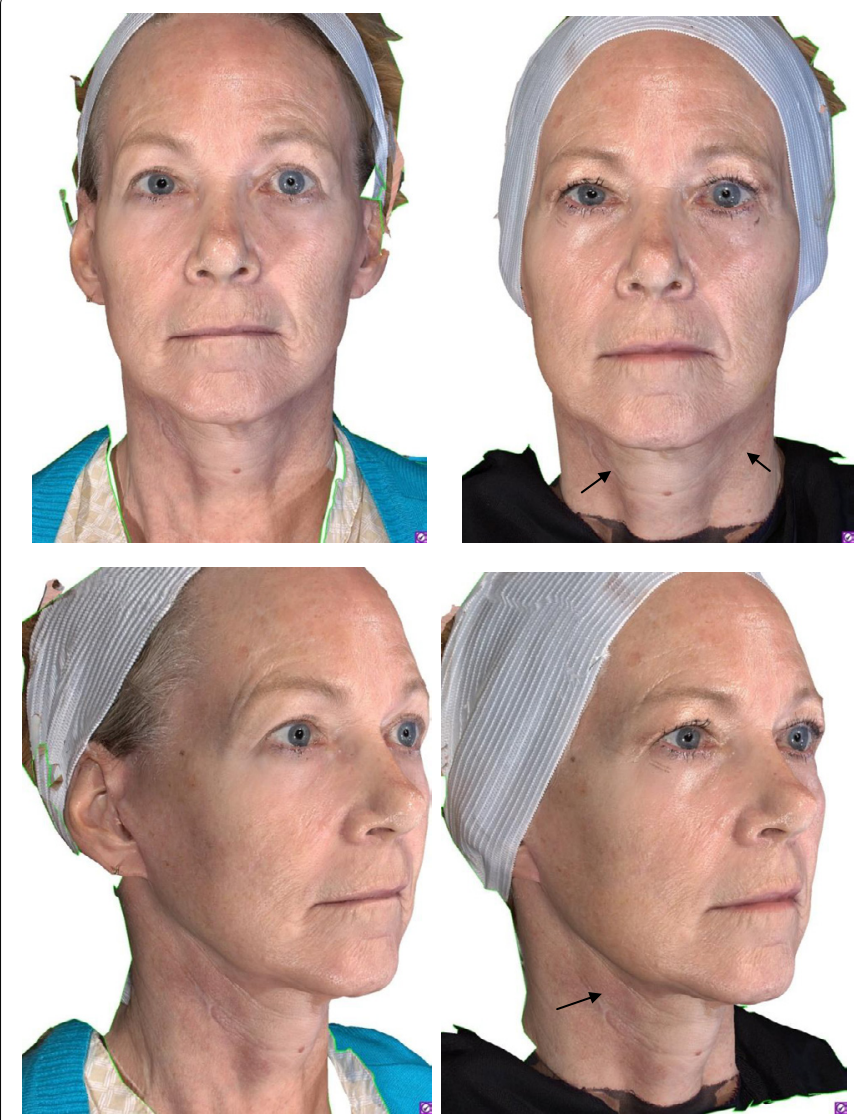

Figure 1: Patient, 60 years old, had Ulthera treatment 10 months after deepSMAS platysmaplasty. Picture on the left shows her before Ulthera treatment and picture on the right shows progress 6 months after Ulthera treatment. No regression of the facial tissues are seen. Improvement can be seen in lower jowls and neck.

Surveys given to patients 24 hours, 1 week, 1 month and 5 months post treatment.

\section{Global Aesthetic Improvement Scale Assessment

Rating Description

1 Very Much Improved- Optimal cosmetic result in this subject

2 Much Improved- Marked improvement in appearance form the initial condition, but not completely optimal for this subject.

3 Improved- Obvious improvement in appearance from initial condition, but a re-treatment is indicated.

4 No Change- The appearance is essentially the same as the original condition.

5 Worse- The appearance is worse than the original condition.

Table 1: Global Aesthetic Improvement Scale Assessment (GAIS). 
Besides our own physician assessments of improvement, an independent board of physicians also graded the patients in a masked assessment. In the masked assessment, the physicians were presented with pre- and post-treatment photos side by side. In order to eliminate bias in the grading, the physicians did not know which photo was the pre-treatment or the post-treatment photo. The physicians would then analyze if there was an improvement between the photos or if there was no change between them. If there was a marked improvement, the physician would then choose which photo was improved. Out of the 9 patients graded, 6 (67\%) were assessed as achieving an improvement. One patient $(11 \%)$ received no change, while two patients $(22 \%)$ received an incorrect assessment.

\section{Discussion}

One of the most prominent issues in aesthetics for both physicians and patients is the maintenance and the integrity of the facelift over time. Of similar interest is the desire to achieve noticeable, positive results in reducing rhytids and increasing elasticity of the skin in the face and neck through non-invasive means. Ultrasound technology has provided a solution to reconcile both of these issues. Although the efficacy of this treatment does not compete with invasive procedures such as rhytidectomy, this technology does have implications for patients who have less pronounced wrinkles or who wish to maintain the quality of a previous facelift over long periods of time.

This study focused on DeepSEE ${ }^{\mathrm{m}}$ ultrasound technology from Ulthera and its ability to maximize the potential of a prior facelift and to maintain the original lift's integrity. Overall, the biggest concern for patients is the pain felt while receiving treatment with Ulthera in spite of the use of topical analgesics. The pain during the procedure could be a contributing factor for those patients who stated that they would not undergo the procedure again. Patients receiving treatment with DeepSEE $^{\text {sa }}$ technology showed moderate improvement in the skin of their face and neck. Based on our patients' own assessment of their results, they noticed modest improvements to their face and neck. The patients' self-assessment was consistent with the physician's assessment at 30 days because each patient had previously undergone a facelift, the moderate change seen after the DeepSEE treatment is satisfactory. The result of this treatment is variable and can be affected by ultrasound settings, skill of the physician and pre- and post-operative care. Limitations to the conclusion of this study include the small sample size and lack of a control sample for comparison. Side effects include blistering or burn, erythema, and contour regulations. The study however, shows there were no adverse events although most patients presented with mild cases of short-term erythema after treatment. Future studies will need to be performed with a larger number of patients in order to maximize the validity of the results.

\section{Conclusion}

DeepSEE $^{m x}$ technology by Ulthera is both safe and effective at maximizing the potential of a facelift as well as maintaining the quality and integrity of the facelift over long periods of time.

\section{References}

1. Alam M, White LE, Martin N, Witherspoon J, Yoo S, et al. (2010) Ultrasound tightening of facial and neck skin: a rater-blinded prospective cohort study. J Am Acad Dermatol 62: 262-269.

2. Dover JS, Zelickson B (2007) 14-Physician Multispecialty Panel. Results of a survey of 5,700 patient monopolar radiofrequency facial skin tightening treatments: assessment of a low-energy multiple-pass technique leading to a clinical end point algorithm. Dermatol Surg 33: 900-907.

3. Alam M, Dover JS (2009) Nonsurgical skin lifting and tightening. London: Elsevier.
4. Mitz V, Peyronie M (1976) The superficial musculo-aponeurotic system (SMAS) in the parotid and cheek area. Plast Reconstr Surg 58: 80-88.

5. Ghassemi A, Prescher A, Riediger D, Axer H (2003) Anatomy of the SMAS revisited. Aesthetic Plast Surg 27:258-264.

6. Har-Shai Y, Sele E, Rubinstien I, Lindenbaum E, Mitz V, et al. (1998) Computerized morphometric quantification of elastin and collagen in SMAS and facial skin and the possible role of fat cells in SMAS viscoelastic properties. Plast Reconstr Surg 102: 2466-2470.

7. Thaller SR, Kim S, Patterson H, Wildman M, Daniller A (1990) The submuscular aponeurotic system (SMAS): a histologic and comparative anatomy evaluation. Plast Reconstr Surg 86: 690-696.

8. Har-Shai Y, Bodner SR, Egozy-Golan D, Lindenbaum ES, Ben-Izhak O et al. (1996) Mechanical properties and microstructure of the superficial musculoaponeurotic system. Plast Reconstr Surg 98: 59-70.

9. Mendelson BC (2001) Surgery of the superficial musculoaponeurotic system: principles of release, vectors, and fixation. Plast Reconstr Surg 107: 1545 1552

10. White M, Makin IRS, Barthe PG, Slayton MH, Gliklich RE (2007) Selective Creation of Thermal injury zones in the superficial musculoaponeurotic system using intense ultrasound therapy: a new target for noninvasive facial rejuvenation. Arch Facial Plast Surg 9: 22-29.

11. Chapelon JY, Ribault M, Vernier F, Souchon R, Gelet A (1999) Treatment of localised prostate cancer with transrectal high intensity focused ultrasound. Eur J Ultrasound 9: 31-38.

12. Foster RS, Bihrle R, Sanghvi NT, Fry FJ, Donohue JP (1993) High-intensity focused ultrasound in the treatment of prostatic disease. Eur Urol 23 Suppl 1: 29-33.

13. Gelet A, Chapelon JY, Bouvier R, Pangaud C, Lasne Y (1999) Local control of prostate cancer by transrectal high intensity focused ultrasound therapy: preliminary results. J Urol 161: 156-162.

14. Gelet A, Chapelon JY, Bouvier R, Souchon R, Pangaud C, et al. (1996) Treatment of prostate cancer with transrectal focused ultrasound: early clinical experience. Eur Urol 29: 174-183.

15. Gianfelice D, Khiat A, Amara M, Belblidia A, Boulanger Y (2003) MR imagingguided focused ultrasound surgery of breast cancer: correlation of dynamic contrast-enhanced MR with histopathologic findings. Breast Cancer Res Treat 82: 93-101.

16. Gianfelice D, Khiat A, Amara M, Belblidia A, Boulanger Y (2003) MR imagingguided focused US ablation of breast cancer: histopathologic assessment of effectiveness-- initial experience. Radiology 227: 849-855.

17. Gianfelice D, Khiat A, Boulanger Y, Amara M, Belblidia A (2003) Feasibility of magnetic resonance imaging-guided focused ultrasound surgery as an adjunct to tamoxifen therapy in high-risk surgical patients with breast carcinoma. $J$ Vasc Interv Radiol 14: 1275-1282.

18. Harari PM, Hynynen KH, Roemer RB, Anhalt DP, Shimm DS, et al. (1991) Development of scanned focussed ultrasound hyperthermia: clinical response evaluation. Int J Radiat Oncol Biol Phys 21: 831-840.

19. Hill CR, ter Haar GR (1995) Review article: high intensity focused ultrasoundpotential for cancer treatment. Br J Radiol 68: 1296-1303.

20. Hutchinson EB, Buchanan MT, Hynynen K (1996) Design and optimization of an aperiodic ultrasound phased array for intracavitary prostate thermal therapies. Med Phys 23: 767-776.

21. Jin CB, Wu F, Wang ZB, Chen WZ, Zhu H (2003) [High intensity focused ultrasound therapy combined with transcatheter arterial chemoembolization for advanced hepatocellular carcinoma]. Zhonghua Zhong Liu Za Zhi 25: 401-403.

22. Kennedy JE, Wu F, ter Haar GR, Gleeson FV, Phillips RR, et al. (2004) Highintensity focused ultrasound for the treatment of liver tumours. Ultrasonics 42 : 931-935.

23. Rowland IJ, Rivens I, Chen L, Lebozer CH, Collins DJ, et al. (1997) MRI study of hepatic tumours following high intensity focused ultrasound surgery. $\mathrm{Br} \mathrm{J}$ Radiol 70: 144-153.

24. Wang ZB (2003) [Clinical application of high-intensity foused ultrasound in obstetrics and gynecology]. Zhonghua Fu Chan Ke Za Zhi 38: 510-512.

25. Watkin NA, Morris SB, Rivens IH, Woodhouse CR, ter Haar GR (1996) A feasibility study for the non-invasive treatment of superficial bladder tumours with focused ultrasound. $\mathrm{Br} \mathrm{J}$ Urol 78: 715-721. 
Citation: Taghizadeh F, Howard AR, Lebowitz A, Ramirez P, Garcia B (2014) Neck Maintenance Post Facelift using Ulthera DeepSEE Technology. Surgery Curr Res 4: 187. doi:10.4172/2161-1076.1000187

26. Wu F, Wang ZB, Chen WZ, Bai J, Zhu H, et al. (2003) Preliminary experience using high intensity focused ultrasound for the treatment of patients with advanced stage renal malignancy. J Urol 170: 2237-2240.

27. Laubach HJ, Makin IR, Barthe PG, Slayton MH, Manstein D (2008) Intense focused ultrasound: evaluation of a new treatment modality for precise microcoagulation within the skin. Dermatol Surg 34: 727-734.

28. Alster TS, Lupton JR (2007) Nonablative cutaneous remodeling using radiofrequency devices. Clin Dermatol 25: 487-491.
29. Dierickx CC (2006) The role of deep heating for noninvasive skin rejuvenation Lasers Surg Med 38: 799-807.

30. Litner JA, Adamson PA (2006) Limited vs extended face-lift techniques: objective analysis of intraoperative results. Arch Facial Plast Surg 8: 186 190

31. Kamer FM, Frankel AS (1998) SMAS rhytidectomy versus deep plane rhytidectomy: an objective comparison. Plast Reconstr Surg 102: 878-881. 